\title{
Study of the Interstellar Medium and Cosmic Rays in local H I Clouds
}

\author{
Tsunefumi Mizuno*on behalf of the Fermi-LAT Collaboration \\ Hiroshima Astrophysical Science Center, Hiroshima University, Higashi-Hiroshima, Hiroshima \\ 739-8526, Japan \\ E-mail: mizunodastro.hiroshima-u.ac.jp
}

\begin{abstract}
Aims. We aim to study the interstellar medium (ISM) and cosmic-rays (CRs) in a mid-latitude region in the third Galactic quadrant (Galactic longitude $l$ from $200^{\circ}$ to $260^{\circ}$ and latitude $|b|$ from $22^{\circ}$ to $60^{\circ}$ ). The region is expected to be dominated by local $\mathrm{H}$ I clouds since it is located at high latitude and free of know large molecular clouds.

Methods. We evaluated the total gas column density $N\left(\mathrm{H}_{\text {tot }}\right)$ by investigating the correlations among $21 \mathrm{~cm}$ survey data (HI4PI), Planck dust thermal emission models (optical depth at $353 \mathrm{GHz} \tau_{353}$ and radiance $R$ ), and Fermi-LAT $\gamma$-ray data in the region studied. In the South region, we first masked areas containing an intermediate velocity cloud or the Orion-Eridanus superbubble, and that with a ratio of the integrated H I $21-\mathrm{cm}$ line intensity to dust emission significantly different from that seen in the rest of the region. We then fit the $\gamma$-ray data with a linear combination of gas template maps based on Planck dust models and other components to obtain the total gas column density $N\left(\mathrm{H}_{\mathrm{tot}, \gamma}\right)$.

Results \& Prospects. We found that $N\left(\mathrm{H}_{\mathrm{tot}, \gamma}\right) / \tau_{353}$ and $N\left(\mathrm{H}_{\mathrm{tot}, \gamma}\right) / R$ depend on dust temperature $T_{\mathrm{d}}$ in the North region, indicating that that neither $\tau_{353}$ nor $R$ were proportional to $N\left(\mathrm{H}_{\mathrm{tot}}\right)$. We also found that $N\left(\mathrm{H}_{\mathrm{tot}, \gamma}\right)$ is not proportional to $\tau_{353}$ but shows non-linear relation in the South region. We will examine the systematic uncertainties and discuss ISM and CR properties inferred from $\gamma$-ray data.
\end{abstract}

7th Fermi Symposium 2017

15-20 October 2017

Garmisch-Partenkirchen, Germany

\footnotetext{
${ }^{*}$ Speaker.
} 